\title{
Perfil de pacientes com neuromielite óptica em tratamento com rituximabe
}

\author{
Profile of patients with neuromyelitis optica in treatment with rituximab \\ Perfil de pacientes con neuromielitis óptica en tratamiento con rituximab
}

Irineu Alves da Silva Júnior ${ }^{1 *}$, Cynthia Angélica Ramos de Oliveira Dourado ${ }^{1}$, Maria Inês Santos da Silva ${ }^{1}$.

\section{RESUMO}

Objetivo: Descrever o perfil de pacientes com neuromielite óptica (NMO) em uso de Rituximabe em um serviço de referência em neurologia. Métodos: Foi realizado um estudo retrospectivo, a partir da análise dos prontuários de 27 pacientes diagnosticados com NMO, em tratamento com rituximabe, atendidos no período de janeiro de 2014 e janeiro 2020. Os dados obtidos foram analisados por estatística descritiva com auxílio do software IBM SPSS 20.0 Resultados: A amostra apresentou idade média de 33,89 $\pm 11,75$ anos, formada por maioria feminina $(88,9 \%)$ e procedente do interior do estado de Pernambuco $(44,4 \%) ;(59,3 \%)$ levou menos de 01 ano para ser diagnosticada e $(55,6 \%)$ faz uso de psicofármacos. Os sintomas de início mais encontrados foram a diminuição da acuidade visual $(70,4 \%)$, a disfunção vesical $(51,9 \%)$ e a paresia $(48,1 \%)$. Apenas $7,4 \%$ desenvolveu reação adversa e o desfecho mais comum foi a continuidade do tratamento (63,0\%). Conclusão: Os resultados evidenciam um perfil clínico muito semelhante ao já relatado na literatura pertinente, contudo observou-se uma tendência de desordens no âmbito da saúde mental que merece mais aprofundamentos.

Palavras-chave: Perfil de saúde, Neuromielite óptica, Rituximabe.

\begin{abstract}
Objective: To describe the profile of patients with neuromyelitis optica (NMO) using rituximab, from a reference service in neurology. Methods: A retrospective study was carried out, based on the analysis of the medical records of 27 patients diagnosed with $\mathrm{NMO}$, undergoing treatment with rituximab, seen between January 2014 and January 2020. The data obtained were analyzed using descriptive statistics with the help of IBM software SPSS 20.0 Results: The sample had a mean age of $33.89 \pm 11.75$ years, formed by a female majority (88.9\%) and from the interior of the state of Pernambuco (44.4\%); (59.3\%) took less than 1 year to be diagnosed and $(55.6 \%)$ used psychiatric drugs. The most common onset symptoms were decreased visual acuity $(70.4 \%)$, bladder dysfunction (51.9\%) and paresis (48.1\%). Only $7.4 \%$ developed an adverse reaction and the most common outcome was continued treatment $(63.0 \%)$. Conclusion: The results show a clinical profile very similar to that already reported in the pertinent literature, however, there was a trend of disorders in the scope of mental health that deserves further study.
\end{abstract}

Key words: Health profile, Neuromyelitis optica, Rituximab.

\section{RESUMEN}

Objetivo: Describir el perfil de pacientes con neuromielitis óptica (NMO) que utilizan rituximab, de un servicio de referencia en neurología. Métodos: Se realizó un estudio retrospectivo, basado en el análisis de los registros médicos de 27 pacientes diagnosticados con NMO, sometidos a tratamiento con rituximab, visto entre enero de 2014 y enero de 2020. Los datos obtenidos se analizaron utilizando estadísticas descriptivas con la ayuda del software de IBM Resultados de SPSS 20.0 Resultados: La muestra tenía una edad promedio de $33.89 \pm 11.75$ años, formada por una mayoría femenina $(88.9 \%)$ y proveniente del interior del estado de Pernambuco (44.4\%), (59.3\%) tomó menos de 01 año a ser diagnosticado y $(55.6 \%)$ usan drogas psicotrópicas. Los síntomas de inicio más comunes fueron disminución de la agudeza visual (70.4\%), disfunción de la vejiga (51.9\%) y paresia (48.1\%). Solo el $7.4 \%$ desarrolló una reacción adversa y el resultado más común fue el tratamiento continuo (63.0\%). Conclusión: Los resultados muestran un perfil clínico muy similar al que ya se informó en la literatura relevante, sin embargo, hubo una tendencia de trastornos en el contexto de la salud mental que merece más estudio.

Palabras clave: Perfil de salud, Neuromielitis óptica, Rituximab.

${ }^{1}$ Universidade de Pernambuco (UPE), Recife - PE. *E-mail: irineujunior9@hotmail.com SUBMETIDO EM: 5/2020 | ACEITO EM: 6/2020 ～PUBLICADO EM: 8/2020 


\section{INTRODUÇÃO}

A neuromielite óptica (NMO, anteriormente chamada de doença de Devic) é uma doença autoimune e desmielinizante do sistema nervoso central, que se manifesta com neurite óptica (NO) e mielite transversa longitudinalmente extensa (MTLE). Atualmente, é reconhecida como uma doença tipicamente recidivante, mas às vezes monofásica, com características clínicas, laboratoriais e de neuroimagem distintas da esclerose múltipla (JASIAK-ZATONSKA M, et al., 2019; TAMBUNAN L, et al., 2019).

Cursa com neuroinflamação autoimune que envolve a medula espinhal, com lesões extensas longitudinalmente, que podem abranger três ou mais segmentos vertebrais, bem como os nervos ópticos, quiasma óptico e partes centrais do cérebro. Para além da apresentação clássica, também pode cursar com lesões cerebrais, diencefálicas ou do tronco encefálico, e soropositividade para o anticorpo antiaquaporina 4, biomarcador sérico da doença (KLEITER I e GOLD R, et al., 2016; TREBST C, et al., 2014; FROTA ERC, et al., 2016).

A caracterização histopatológica apresenta dano astrocítico autoimune, seguido de desmielinização, perda neuronal e necrose frequentemente pronunciada, com perda seletiva e precoce de aquaporina 4 , uma proteína com função de canal de água, localizada na barreira hematoencefálica do SNC, envolvida na homeostase de fluidos, que culmina no processo inflamatório local e consequente lesão nervosa (TREBST C, et al., 2014; FROTA ERC, et al., 2016).

Ainda é desconhecida a etiologia, porém sabe-se que possui um curso autoimune, e que também pode estar associada a infecções virais e/ou síndromes pós-virais, inflamação de tecidos contíguos (meninges, órbita e seios da face) e doenças sistêmicas, como a sarcoidose, a sífilis, a tuberculose e a doença de Lyme (CHAVES MLF, et al., 2009).

É reconhecida como uma patologia rara, caracterizada por afetar até 65 pessoas para cada 100.000 indivíduos, com uma prevalência de 1 a 4,4 para cada 100.000 pessoas no mundo ocidental (FABRIZZIO GC, et al., 2018). O tratamento visa controlar o dano inflamatório causado pelos ataques agudos através de corticoidoterapia ou plasmaférese, e evitar recaídas por meio de corticosteroides associados a imunossupressores.

Vale salientar que a administração da terapia intravenosa se dá por meio de pulsoterapia, que consiste na infusão de altas doses de medicações em curto período de tempo (TRADTRANTIP L, et al., 2020). Os casos de refratariedade, em que não há resposta satisfatória ao imunossupressor prescrito na dose e duração apropriada, estão frequentemente associados a um prognóstico desfavorável e requerem com urgência outra linha de tratamento (COLLONGUES N, et al., 2015).

Neste sentido, o rituximabe se traduz como um tratamento promissor na NMO, comumente utilizado em casos de falha terapêutica, por se tratar de um anticorpo monoclonal, antineoplásico e antirreumático, utilizado para tratar neoplasias linfoides derivadas de células B e doenças autoimunes mediadas por anticorpos. Por outro lado, seu uso predispõe o indivíduo a reações adversas distintas e quadros infecciosos de repetição, devido a sua ação imunossupressora (GAO F, et al., 2019).

O prognóstico da NMO está relacionado à frequência e à gravidade de recorrência dos surtos, estimado em $60 \%$ no primeiro ano e em $90 \%$ nos três anos subsequentes. A incapacidade motora e visual geralmente resulta do efeito cumulativo de recaídas. $O$ tratamento eficaz do surto, seguido pela prevenção de recidivas, é considerado a chave para a redução da incapacidade na NMO (FROTA ERC, et al., 2016; ABBOUD, et al., 2015).

Diante do exposto, justifica-se este estudo pela necessidade de dimensionar a problemática da NMO, enquanto doença crônica degenerativa, que afeta diretamente a produtividade e a qualidade de vida, com abordagem terapêutica diversificada, e que tem o rituximabe como o tratamento mais promissor.

A relevância do estudo também vai de encontro com a escassa produção de conhecimento sobre o perfil de pacientes acometidos pela neuromielite óptica no Brasil. Na literatura, as pesquisas com foco em NMO detêm-se à descrição de cuidados e relatos de casos. Assim, este estudo teve como objetivo descrever 0 perfil de pacientes com neuromielite óptica em uso de rituximabe. 


\section{MÉTODOS}

Trata-se de um estudo retrospectivo, descritivo e quantitativo, realizado em março de 2020 no serviço de neurologia de um hospital de referência em uma cidade no estado de Pernambuco (PE). Foram revisados os prontuários de 27 pacientes $(n=27)$ com diagnóstico de NMO, cadastrados no serviço de pulsoterapia da referida instituição para uso de Rituximabe. Desta forma, foram considerados como critérios de inclusão pacientes com: idade acima de 18 anos; de ambos os sexos; atendidos no período de janeiro de 2014 a janeiro de 2020.

A coleta de dados foi realizada no mês de março de 2020 por meio de prontuário eletrônico, e auxílio de instrumento de coleta semiestruturado elaborado pelos pesquisadores. A análise contemplou fichas de esclarecimento, resumos de alta hospitalar e evoluções clínicas. As variáveis analisadas foram: sexo, idade, estado civil, procedência, comorbidades, tempo para diagnóstico, sintomas de início, tratamentos prévios, tempo de rituximabe, reações adversas e os desfechos mais comuns da terapêutica supracitada.

Para avaliar a distribuição das variáveis quantitativas categóricas, foram calculadas as frequências absolutas e relativas (percentuais) de cada variável. Foi utilizado o teste de qui-quadrado $\left(X^{2}\right)$ para comparação das distribuições das proporções de cada categoria. O nível de significância pré-fixado foi de $5,0 \%(p<0,05)$ e os testes estatísticos foram realizados no software Statistical Package for Social Science (SPSS) 20.0.

Em relação aos aspectos éticos em pesquisa, o estudo respeitou a Resolução 466/2012 do Conselho Nacional de Saúde e foi aprovado pelo Comitê de Ética em Pesquisa do Hospital da Restauração Gov. Paulo Guerra, sob o número de Certificado de Apresentação para Apreciação Ética (CAAE) 30121120.1.0000.5198 e parecer 3.965.825.

\section{RESULTADOS}

A Tabela 1 representa os dados da distribuição do perfil sociodemográfico dos pacientes portadores de Neuromielite Óptica analisados nesta pesquisa. Verifica-se que a maioria é do sexo feminino $(88,9 \%)$, possui idade abaixo de 40 anos $(74,0 \%)$, procedente do interior do estado de PE $(44,4 \%)$ e solteiros $(63,0 \%)$.

As variáveis sexo e procedência foram significativas para o teste de comparação de proporções, indicando que o grupo feminino representa a variável sexo e que a procedência do interior de PE representa a maior fração da amostra analisada. Já acerca das variáveis idade e estado civil, o teste indica que não há diferença entre as categorias de cada variável.

\begin{tabular}{|c|c|c|c|}
\hline Fator avaliado & $\mathbf{N}$ & $\%$ & p-valor ${ }^{1}$ \\
\hline \multicolumn{4}{|l|}{ Sexo } \\
\hline Masculino & 03 & 11,1 & \multirow{2}{*}{$<0,001$} \\
\hline Feminino & 24 & 88,9 & \\
\hline \multicolumn{4}{|l|}{ Idade } \\
\hline Até 25 anos & 10 & 37,0 & \multirow{5}{*}{0,717} \\
\hline 26 a 40 anos & 10 & 37,0 & \\
\hline$>40$ anos & 07 & 25,9 & \\
\hline Mínimo - Máximo & & $17-61$ & \\
\hline Média \pm Desvio padrão & & $33,89 \pm 11,75$ & \\
\hline \multicolumn{4}{|l|}{ Procedência } \\
\hline Recife - PE & 08 & 29,6 & \multirow{4}{*}{0,026} \\
\hline Reg. Metropolitana Recife & 06 & 22,2 & \\
\hline Interior de PE & 12 & 44,4 & \\
\hline Outro estado & 01 & 3,7 & \\
\hline \multicolumn{4}{|l|}{ Estado Civil } \\
\hline Solteiro & 17 & 63,0 & \multirow{2}{*}{0,178} \\
\hline Casado & 10 & 37,0 & \\
\hline
\end{tabular}

Legenda: ${ }^{1}$ Qui-quadrado de proporção.

Fonte: Júnior IAS, et al., 2020. 
Na Tabela 2 temos a distribuição de uso de psicofármacos. Verifica-se que a maioria $(55,6 \%)$ faz uso de medicamento controlado. Dos que fazem uso, os mais frequentes são o ansiolítico $(25,9 \%)$ e o antidepressivo $(25,9 \%)$. Não foi observada diferença estatística significativa em nenhuma variável.

Tabela 2 - Distribuição do uso e grupos de psicofármacos na amostra pesquisada.

\begin{tabular}{cccc}
\hline Fator avaliado & N & $\%$ & p-valor $^{1}$ \\
\hline Consumo & & & \\
\hline Sim $^{*}$ & 15 & 55,6 & \multirow{2}{*}{0,564} \\
Não & 12 & 44,4 & \\
\hline Classe farmacológica* & & & \\
\hline Ansiolíticos & 07 & 25,9 & \\
Antidepressivos & 07 & 25,9 & 0,091 \\
Antipsicóticos & 01 & 3,7 & \\
\hline
\end{tabular}

Legenda: ${ }^{1}$ Qui-quadrado de proporção. *Variáveis relacionadas

Fonte: Júnior IAS, et al., 2020.

$\mathrm{Na}$ Tabela 3 temos a distribuição da sorologia para anti-aquaporina 4 (antiAQP4), bem como do histórico de recorrência de surtos e do desenvolvimento de reações adversas decorrentes do tratamento com 0 rituximabe. Verifica-se que a maioria possui sorologia reagente para o anticorpo AQP4 (55,6\%), e que toda a amostra (100\%) apresenta a forma recidivante de apresentação clínica. Além disso, o desenvolvimento de reação adversa esteve presente em $(7,4 \%)$ da amostra, que teve como reações a pancitopenia $(3,7 \%)$ e 0 broncoespasmo $(3,7 \%)$. Vale ressaltar que não houve relato de dados acerca da existência de reação adversa, em $88,9 \%$ da amostra. Não foi encontrada significância estatística nas variáveis apresentadas.

Tabela 3 - Distribuição dos dados acerca da sorologia para anticorpo aquaporina 4 , recorrência de surtos e desenvolvimento de reações adversas na amostra pesquisada.

\begin{tabular}{cccc}
\hline Fator avaliado & $\mathbf{n}$ & $\%$ & p-valor $^{1}$ \\
\hline Anti-aquaporina 4 (AQP4) & & & \\
\hline Reagente & 15 & 55,6 & \multirow{2}{*}{0,564} \\
Não-reagente & 12 & 44,4 & \\
\hline Forma de apresentação clínica & & & \\
\hline Recidivante & 27 & 100 & - \\
Monofásica & 00 & 00 & \\
\hline $\begin{array}{c}\text { Desenvolvimento de reação adversa } \\
\text { secundária ao Rituximabe }\end{array}$ & & \\
\hline Sim ${ }^{* *}$ & 02 & 7,4 & \\
Não & 01 & 3,7 & 0,564 \\
Omissos* & 24 & 88,9 & \\
\hline Reação adversa apresentada** & & & \\
\hline Pancitopenia & 01 & 3,7 & \multirow{2}{*}{1,000} \\
Broncoespasmo & 01 & 3,7 & \\
\hline
\end{tabular}

Legenda: ${ }^{1}$ Qui-quadrado de proporção. *Dados não relatados ${ }^{* *}$ Variáveis relacionadas

Fonte: Júnior IAS, et al., 2020.

Na Tabela 4 temos a caracterização das comorbidades e a distribuição dos desfechos clínicos mais comuns. Verifica-se que a maioria não apresenta comorbidades $(77,8 \%)$ e dentre os que apresentam $(22,2 \%)$, as doenças cardiovasculares se mostraram mais frequentes (18,5\%). Em relação aos desfechos, verifica-se também que a maioria $(63,0 \%)$ deu continuidade ao tratamento, e que $(11,1 \%)$ da amostra foi a óbito. Dos que foram a óbito, apenas $(3,7 \%)$ teve causa esclarecida, enquanto $(7,4 \%)$ não teve a causa informada. $O$ teste indica que para as variáveis comorbidade e desfecho clínico, os maiores percentuais são significativamente diferentes dos menores, já para o tipo de comorbidade e a causa do óbito, não houve diferença entre as categorias, indicando homogeneidade. 
Tabela 4 - Caracterização das comorbidades e desfecho clínico da amostra estudada.

\begin{tabular}{cccc}
\hline Fator avaliado & $\mathbf{n}$ & $\%$ & p-valor $^{1}$ \\
\hline Comorbidades & & & \\
\hline Sim & 06 & 22,2 & \multirow{2}{*}{0,004} \\
Não & 21 & 77,8 & \\
\hline Comorbidades apresentadas & & & \\
\hline Doença cardiovascular & 05 & 18,5 & \\
Disfunção tireoidiana & 01 & 3,7 & 0,102 \\
$\quad$ Omissos* & 21 & 77,8 & \\
\hline Desfecho clínico & & & \\
\hline Continua o tratamento & 17 & 63,0 & \\
Abandonou o tratamento & 07 & 25,9 & 0,041 \\
$\quad$ Óbito** & 03 & 11,1 & \\
\hline Causa do óbito** & & & \\
\hline Choque séptico & 01 & 3,7 & \multirow{2}{*}{0,564} \\
Não informado & 02 & 7,4 & \\
\hline
\end{tabular}

Legenda: ${ }^{1}$ Qui-quadrado de proporção. *Dados não relatados **Variáveis relacionadas.

Fonte: Júnior IAS, et al., 2020.

Na Tabela 5 temos a distribuição dos tratamentos realizados anteriormente ao rituximabe. Verifica-se que a maioria já fez uso de azatioprina $(81,5 \%)$ e de pulsoterapia com metilprednisolona $(59,3 \%)$. A plasmaférese $(44,4 \%)$ e a pulsoterapia com imunoglobulina $(25,9 \%)$ foram as propostas terapêuticas menos utilizadas nesta amostra. O teste de comparação de proporção indica que na amostra pesquisada, o tratamento com a azatioprina foi prevalente, já para a pulsoterapia com imunoglobulina a maior prevalência foi a de não tratamento e para a metilprednisona e plasmaférese não houve diferença estatística em fazer ou não.

Tabela 5 - Distribuição das terapias prévias ao uso do Rituximabe na amostra pesquisada.

\begin{tabular}{cccc}
\hline Fator avaliado & N & $\%$ & p-valor $^{1}$ \\
\hline Uso de azatioprina & & & \\
\hline Sim & 22 & 81,5 & \multirow{2}{*}{0,001} \\
Não & 05 & 18,5 & \\
\hline Pulsoterapia com & & & \\
Imunoglobulina & & & \\
\hline Sim & 07 & 25,9 & \multirow{2}{*}{0,012} \\
Não & 20 & 74,1 & \\
\hline Pulsoterapia com & & & \\
Metilprednisolona & & & \\
\hline Sim & 16 & 59,3 & 0,336 \\
Não & 11 & 40,7 & \\
\hline Plasmaférese & & & \\
\hline Sim & 12 & 44,4 & 0,564 \\
Não & 15 & 55,6 &
\end{tabular}

Legenda: ${ }^{1}$ Qui-quadrado de proporção.

Fonte: Júnior IAS, et al., 2020.

Já na Tabela 6 temos a distribuição dos sintomas de início. Verifica-se que os mais frequentes são a diminuição da acuidade visual $(70,4 \%)$, a disfunção vesical $(51,9 \%)$ e a paresia $(48,1 \%)$. Vale destacar também, que os sintomas menos prevalentes foram a constipação $(25,9 \%)$, a parestesia $(25,9 \%)$ e a plegia $(29,6 \%)$. Além de que os sintomas que demonstraram significância estatística $(p$-valor $<0,05)$ foram a diminuição de acuidade visual, a constipação, a parestesia e a plegia, com diferença significativa para o maior percentual. 
Tabela 6 - Distribuição dos Sinais e Sintomas prévios ao diagnóstico na amostra estudada.

\begin{tabular}{|c|c|c|c|}
\hline Fator avaliado & $\mathbf{N}$ & $\%$ & p-valor ${ }^{1}$ \\
\hline \multicolumn{4}{|l|}{ Diminuição de acuidade visual } \\
\hline Sim & 19 & 70,4 & \multirow{2}{*}{0,034} \\
\hline Não & 08 & 29,6 & \\
\hline \multicolumn{4}{|l|}{ Disfunção vesical } \\
\hline Sim & 14 & 51,9 & \multirow[b]{2}{*}{0,847} \\
\hline Não & 13 & 48,1 & \\
\hline \multicolumn{4}{|l|}{ Constipação } \\
\hline Sim & 07 & 25,9 & \multirow{2}{*}{0,012} \\
\hline Não & 20 & 74,1 & \\
\hline \multicolumn{4}{|l|}{ Parestesia } \\
\hline Sim & 08 & 29,6 & \multirow{3}{*}{0,027} \\
\hline Não & 17 & 63,0 & \\
\hline Omissos* & 02 & 7,4 & \\
\hline \multicolumn{4}{|l|}{ Membros acometidos } \\
\hline Membros inferiores (MMII) & 03 & 11,1 & \multirow{5}{*}{0,666} \\
\hline Membros superiores (MMSS) & 01 & 3,7 & \\
\hline Ambos (MMII e MMSS) & 02 & 7,4 & \\
\hline Hemicorpo & 01 & 3,7 & \\
\hline Omissos* & 20 & 74,1 & \\
\hline \multicolumn{4}{|l|}{ Paresia } \\
\hline Sim & 13 & 48,1 & \multirow{2}{*}{0,847} \\
\hline Não & 14 & 51,9 & \\
\hline \multicolumn{4}{|l|}{ Membros acometidos } \\
\hline Membros inferiores (MMII) & 05 & 18,5 & \multirow{5}{*}{0,691} \\
\hline Membros superiores (MMSS) & 02 & 7,4 & \\
\hline Ambos (MMII e MMSS) & 03 & 11,1 & \\
\hline Hemicorpo & 03 & 11,1 & \\
\hline Omissos* & 14 & 51,9 & \\
\hline \multicolumn{4}{|l|}{ Plegia } \\
\hline Sim & 08 & 29,6 & \multirow{2}{*}{0,034} \\
\hline Não & 19 & 70,4 & \\
\hline \multicolumn{4}{|l|}{ Membros acometidos } \\
\hline Inferiores (paraplegia) & 05 & 18,5 & \multirow{3}{*}{0,480} \\
\hline Ambos (tetraplegia) & 03 & 11,1 & \\
\hline Omissos* & 19 & 70,4 & \\
\hline
\end{tabular}

Legenda: ${ }^{1}$ Qui-quadrado de proporção. * Dados não relatados

Fonte: Júnior IAS, et al., 2020.

Em relação a distribuição do tempo para o diagnóstico e tempo com a doença e ao uso do Rituximabe, observa-se que a maioria da amostra levou menos de 01 ano para ser diagnosticada $(59,3 \%),(14,8 \%) 2$ anos e (3,7\%) da amostra para 1 ano, 3 anos, 5 anos, 7 anos, 11 anos, 12 anos e 20 anos. Verifica-se, ainda, que (51,9\%) possuem 6 anos de diagnóstico da doença, em sequência, (22,2\%) 3 anos, $(14,8 \%) 4$ anos, $(7,4 \%)$ 5 anos e (3,7\%) 1 ano. No que diz respeito ao tempo de uso do Rituximabe, $(37,0 \%)$ da amostra possui 1 ano de uso da medicação, (18,5\%) possuem 2, 3 e 6 anos e $(7,4 \%) 4$ anos.

\section{DISCUSSÃO}

A amostra composta por dados de 27 pacientes acompanhados em um centro especializado de atendimento a afecções neurológicas, buscou analisar o perfil de pacientes portadores de neuromielite óptica em tratamento com o Rituximabe, por meio da descrição das características sociodemográficas e clínicas. As variáveis sociodemográficas sexo e idade se mostraram compatíveis com os dados da literatura. As características da amostra estudada apresentaram idade média da doença inferior às relatadas na literatura. 
De acordo com Frota ERC, et al. (2016), a NMO é mais comum em mulheres (na proporção de oito mulheres para cada homem) e tende a iniciar em faixa etária mais tardia, com média de idade entre 35 e 40 anos. Também há estudos semelhantes que apontam ser de três a cinco vezes mais frequente no sexo feminino, com início, em média, em torno de 32,6 a 45,7 anos (KOUDRIAVTSEVA T e RENNA R, 2015; BUI M, et al., 2019; FABRIZZIO GC, et al., 2018; TREBST C, et al., 2014; BIBIANO AMB, et al., 2015).

Não foram encontrados na literatura dados para discussão das variáveis "procedência" e "estado civil", dada a escassez de estudos descritivos com esta temática. Porém, foi possível observar que a maioria $(44,4 \%)$ é proveniente do interior do estado. Para Sarva H, et al. (2014), os pacientes tratados em hospitais menos privilegiados podem ter pior prognóstico, que pode ser devido em parte a atrasos no diagnóstico e no encaminhamento para clínicas especializadas, que comumente se encontram nas grandes cidades. No tocante ao estado civil, Neves CFS, et al. (2017) apontam que embora os pacientes casados tenham a família, e sobretudo o cônjuge, como rede de apoio, isso não significa que os indivíduos solteiros não possuam com suporte familiar ou de amigos.

Ao analisar o consumo de psicofármacos, os estudos revelam que os medicamentos psicotrópicos têm sido prescritos com frequência para tratar sintomas neuropsiquiátricos como a ansiedade, o estresse e sintomas depressivos frente às limitações da vida diária, causadas pelo adoecimento. Neste sentido, o tratamento inclui, sobretudo, o uso dos ansiolíticos, que têm como principal representante desta classe os benzodiazepínicos, e os antidepressivos, por contribuir no controle das emoções, do humor e do comportamento (AJMERA MR, et al., 2017; GERRITSEN AAJ, et al., 2019; FÁVERO VR, et al., 2017; FABRIZZIO GC, et al., 2018; LIU Y e TANG X, 2018).

A azatioprina se mostra na literatura, como o imunossupressor de uso mais frequente, haja vista sua facilidade de administração, por via oral, e sua capacidade de reduzir a taxa de recaídas, ou seja, de diminuir a ocorrência de surtos, e ajudar a melhorar as queixas de ordem visual. Além disso, quando a resposta ao tratamento é ausente ou há efeitos colaterais, a dose é ajustada ou, se necessário, um tratamento diferente costuma ser aplicado (HUANG T, et al., 2018; TREBST C, et al., 2014).

Também foram encontrados estudos que corroboram a alta frequência de uso da pulsoterapia com corticosteroides, em decorrência da sua indicação para tratamento do surto, e objetiva minimizar a perda axonal no estágio agudo. Assim, qualquer exacerbação deve ser tratada prontamente como surto por meio de pulsoterapia com metilprednisolona por 3 a 5 dias. Contudo, a ausência de resposta a este fármaco pode ocorrer próximo de $20 \%$ na fase aguda, e os casos refratários podem responder à plasmaférese (HUANG T, et al., 2018; FROTA ERC, et al., 2016).

A literatura menciona que em caso de resposta insatisfatória à terapia com esteroides, pode-se recorrer à terapia de troca de plasma, que tem o objetivo de reduzir fatores humorais, e particularmente a carga de antiaquaporina 4 circulante, por meio de ciclos que variam entre 5 a 7 sessões em dias alternados, para prevenir e tratar ataques severos (FROTA ERC, et al., 2016; KLEITER I e GOLD R, 2016). De acordo com Trebst C, et al. (2014), a pulsoterapia com imunoglobulina também é sugerida como alternativa para pacientes que possuem contraindicação a um dos tratamentos mencionados e, principalmente, em crianças, pois tem demonstrado boa tolerância e eficácia neste público (TREBST C, et al., 2014; GOUDOURIS ES, et al., 2017).

$O$ rituximabe se caracteriza como um anticorpo monoclonal, utilizado em caso de falha terapêutica às modalidades mencionadas, ou como primeira linha para reduzir a gravidade de recorrência da doença. Foi inicialmente projetado para uso em linfoma não-Hodgkin, mas tem se mostrado vantajoso no tratamento de distúrbios reumatológicos e neurológicos autoimunes, sobretudo a NMO. A literatura aponta, ainda, que a remissão gradativa dos sintomas se traduz como o principal benefício da medicação, além da diminuição da ocorrência de surtos e melhora da incapacidade na maioria dos pacientes. (GAO F, et al., 2019; ETEMADIFAR M, et al., 2017).

Em relação ao tempo de uso, a adesão ao tratamento costuma ser eficaz, visto que o rituximabe apresenta tolerância aceitável. De acordo com os estudos revisados, a depleção de células B após a pulsoterapia com rituximabe dura, em média, 12 meses no sangue periférico antes da geração de uma nova população de linfócitos $B$ e, quão logo é iniciado ou reinfundido, ajuda a diminuir a possibilidade de reexpansão destas células e, consequentemente, de surtos da doença (DAMATO V, et al., 2016; GAO F, et al., 2019). 
O desenvolvimento de reações adversas secundárias à pulsoterapia com o rituximabe se mostrou frequente na literatura. Uma revisão sistemática realizada por Etemadifar M, et al. (2017), revelou que todos os estudos analisados relataram efeitos adversos na terapia com rituximabe em pacientes com NMO. Contudo, a maioria deles, quando existentes, foi quase transitória. Contudo, a literatura caracteriza o broncoespasmo como uma reação de natureza anafilática pós-infusão, e um distúrbio respiratório adverso incomum. Em contrapartida, a pancitopenia é reportada como uma reação adversa hematológica comum, que pode requerer, inclusive, transfusão de sangue e plaquetas. (GAO F, et al., 2019; SIMÕES M, et al., 2019; ROCHE, 2019).

A recorrência de surtos também se mostrou frequente na literatura. Segundo Kim EB, et al., (2019), o curso clínico da NMO pode ser monofásico ou recidivante. Na apresentação monofásica, que se caracteriza pela ocorrência de um único quadro agudo, a sintomatologia tende a ser mais grave que a recorrente, porém com boa recuperação. Já a forma recorrente consiste na ocorrência recidivante de quadros agudos, que fornecem um prognóstico ruim, visto que mais da metade dos pacientes desenvolve severa perda visual e dificuldade de locomoção.

Embora a eficácia e segurança da medicação ainda não tenham sido totalmente estabelecidas, a continuidade do tratamento é observada em boa parte dos estudos conduzidos com o rituximabe, dada a remissão dos sintomas, seja em monoterapia ou pulsoterapia combinada (GAO F, et al., 2019; ETEMADIFAR M, et al., 2017; TREBST C, et al., 2014).

De acordo com Gao F, et al. (2019), os óbitos associados ao tratamento com rituximabe, decorrem da diminuição dos níveis de imunoglobulina, o que predispõe ao risco de infecção e septicemia. Vale destacar que uma revisão sistemática conduzida por Damato $\mathrm{V}$, et al. (2016) identificou que a NMO encontra-se associada a uma taxa de mortalidade de até $12 \%$. A coexistência de disfunção tireoideana com a NMO foi corroborada por meio de um estudo realizado por Pitock A e Lucchinetti C (2015), que descreveu o hipotireoidismo como uma condição autoimune comumente encontrada neste tipo de agravo.

Um estudo conduzido por Ajmera MR, et al. (2017) identificou a hipertensão arterial como a comorbidade sistêmica mais prevalente entre os casos de NMO analisados, corroborando assim os achados desta pesquisa. Todavia, as evidências existentes sugerem que as condições mais comuns que coexistem com a NMO são doenças autoimunes, como o lúpus eritematoso sistêmico, a miastenia gravis e, mais frequentemente, a artrite reumatoide (AMORIM ALM, et al., 2017; AJMERA MR, et al., 2017).

No que diz respeito ao status sorológico de anti-aquaporina 4, os estudos indicam que os títulos do anticorpo podem flutuar ao longo da doença, até mesmo com sua negativação relacionada ao uso prévio de imunoterapias, como a pulsoterapia com metilprednisolona, por exemplo. Porém, vale salientar que a detecção sorológica de antiaquaporina 4 prevê recaídas de mielite e neurite óptica com incapacidade neurológica, e justifica o início imediato da terapia imunossupressora (FROT ERC, et al., 2016; PITOCK S e LUCCHINETTI C, 2015).

Em relação ao tempo para diagnóstico, verifica-se que os pacientes que custam a ser diagnosticados apresentam maior taxa de incapacidade motora e neuroplasticidade mais severamente reduzida, fato este que reforça o caráter degenerativo da doença. Diversos estudos indicam que, como os sintomas da NMO são incapacitantes, e sobretudo os de ordem visual, a busca pelo serviço de saúde, não necessariamente especializado, acontece de forma precoce (HUDA S, et al., 2019; ZHANG Y, et al., 2020).

A literatura refere que o sintoma inicial mais comum é o envolvimento do nervo óptico, uma condição dolorosa que se agrava com o movimento, e é comumente acompanhada de diminuição da acuidade visual no olho afetado. Geralmente, após cinco anos, cerca de 50\% dos pacientes apresentam perda da visão, uni ou bilateralmente, e incapacidade de deambular sem auxílio, com algum grau de disfunção esfincteriana, parestesia e insuficiência respiratória (ZAREI S, et al., 2018; WOO S, et al., 2016; FROTA ERC, et al., 2016).

A perda motora e sensorial, principalmente de membros inferiores, decorre do acometimento da medula espinhal, e se caracteriza como mielite transversa completa, geralmente com paraparesia ou tetraparesia, e nível sensitivo quase sempre simétrico. A disfunção esfincteriana, por sua vez, surge devido a lesões que 
afetam as vias neurais do sistema genital, e o desenvolvimento de sintomas como fadiga, espasmos dolorosos, e disfunção vesical e/ou intestinal (ZHANG Y, et al., 2020; LANA-PEIXOTO MA e TALIM N, 2019; ZAREI S, et al., 2018; FROTA ERC, et al., 2016).

No que diz respeito à paresia e à plegia, Huda S, et al. (2019) apontam que a MLTE pode evoluir para paraplegia ou tetraplegia, a depender do nível da medula envolvido, corroborando assim os achados desta pesquisa. Além disso, os autores descrevem como frequente a necessidade de irrigação intestinal regular para controle da constipação nestes pacientes, e do uso de auto cateterismo intermitente nos distúrbios vesicais.

As alterações sensoriais que constituem a parestesia, a nível de mielite transversa, juntamente com as parestesias e plegias descritas, apontam para o potencial incapacitante da NMO, o que justifica a necessidade de abordagem terapêutica eficaz, a fim de reduzir a rápida progressão da mesma (HUDA S, et al., 2019; FROTA ERC, et al., 2016).

Como limitação encontrada ao longo do estudo, destaca-se a escassez de dados epidemiológicos nacionais que pudessem proporcionar uma aproximação mais fidedigna com o tema. Contudo, vale considerar que a pesquisa em bases internacionais forneceu subsídios para a compreensão do perfil proposto, e na construção de um trabalho exploratório na região nordeste, propiciando uma assistência mais direcionada e pautada na doença e em suas especificidades clínicas. Espera-se que o estudo contribua com a comunidade científica, de modo a servir de referência na condução de futuras pesquisas.

\section{CONCLUSÃO}

O estudo possibilitou um panorama acerca do atual perfil de pacientes com neuromielite óptica em tratamento com rituximabe. Em sua maioria, o perfil é composto por mulheres jovens, solteiras, provenientes do interior do estado, que buscam o serviço especializado em função de um quadro clínico incapacitante, caracterizado por diminuição de acuidade visual, disfunção vesical e paraparesia, associado a sorologia reagente para antiaquaporina 4; que fazem uso de psicofármacos e que dão prosseguimento ao tratamento para minimizar as recidivas e possíveis complicações clínicas. Os achados deste estudo reforçam a importância do diagnóstico e do início precoce da terapia imunossupressora.

\section{REFERÊNCIAS}

1. ABBOUD H, et al. Treatment of acute relapses in neuromyelitis optica: Steroids alone versus steroids plus plasma exchange. Multiple Sclerosis Journal, 2015; 22(2): 185-192.

2. AJMERA MR, et al. Evaluation of comorbidities and health care resource use among patients with highly active neuromyelitis optica. Journal of the Neurological Sciences, 2017; 384:96-103.

3. AMORIM ALM, et al. Associação entre doença desmielinizante e doença reumática autoimune em uma população pediátrica. Revista Brasileira de Reumatologia, 2017; 57(3):224-228.

4. BIBIANO AMB, et al. Functional capacity in the Devic disease: case report. Revista Neurociências, 2015; 23(4): 603608.

5. BUI M, et al. Neuromyelitis Optica Spectrum Disorder: A Rare Case of Isolated Brainstem Syndrome. Cureus, 2019; 11(9): 01-07.

6. CHAVES MLF, et al. Rotinas em neurologia e neurocirurgia. Porto Alegre: Artmed, 2009. 861p.

7. COLLONGUES N, et al. Efficacy of rituximab in refractory neuromyelitis optica. Multiple Sclerosis Journal, 2015; 22(7): 955-959.

8. DAMATO V, et al. Efficacy and Safety of Rituximab Therapy in Neuromyelitis Optica Spectrum Disorders. JAMA Neurology, 2016; 73 (11): 1342-1348.

9. ETEMADIFAR M, et al. Eficácia e segurança do rituximabe na neuromielite óptica: revisão das evidências. Journal of Research in Medical Sciences, 2017; 22(18): 01-15.

10. FABRIZZIO GC, et al. Care management of a patient with Devic's Disease in Primary Health Care. Revista da Escola de Enfermagem da USP, 2018; 52: 01-07.

11. FÁVERO VR, et al. Uso de ansiolíticos: abuso ou necessidade? Revista Visão Acadêmica, 2017; 18(4): 98-106.

12. FROTA ERC, et al. Recomendações no tratamento da Esclerose Múltipla e Neuromielite Óptica. Departamento Científico de Neuroimunologia da ABN. São Paulo: Editora e Eventos Omnifarma, 2016; 232p.

13. GAO F, et al. Effectiveness of Rituximab in Neuromyelitis Optica: A Meta-Analysis. BMC Neurology, $2019 ; 19(1): 36$.

14. GERRITSEN AAJ, et al. Psychotropic drug use in community-dwelling people with young-onset dementia: two-year course and determinants. Aging \& Mental Health, 2019; 20: 01-08. 
15. GOUDOURIS ES, et al. II Consenso Brasileiro sobre o uso de imunoglobulina humana em pacientes com imunodeficiências primárias. Einstein, 2017; 15(1): 1-16

16. HUANG T, et al. Treatment strategies for neuromyelitis optica. Tzu Chi Medical Journal, 2018: 30(4): 204-208.

17. HUDA S, et al. Neuromyelitis optica spectrum disorders. Clinical Medicines, 2019; 19 (2): 169-176.

18. JASIAK-ZATONSKA M, et al. The Immunology of Neuromyelitis Optica - Current Knowledge, Clinical Implications, Controversies and Future Perspectives. International journal of Molecular Sciences, 2016; 17 (3): 273.

19. KIM WB, et al. Rehabilitation of neuromyelitis optica: Two CARE-compliant case reports. Medicine, 2019; 98(41): 0105.

20. KLEITER I, GOLD R. Present and Future Therapies in Neuromyelitis Optica Spectrum Disorders. Neurotherapeutics, 2016; 13(1): 70-83.

21. KOUDRIAVTSEVA T, RENNA R. Profile of neuromyelitis optica and spectrum disorders in an Indian cohort. Journal of Neurosciences in Rural Practice, 2015; 6(3): 295-296.

22. LANA-PEIXOTO MA, TALIM N. Neuromyelitis Optica Spectrum Disorder and Anti-MOG Syndromes. Biomedicines, 2019; 7(2): 42.

23. LIU Y, TANG X. Depressive Syndromes in Autoimmune Disorders of the Nervous System: Prevalence, Etiology, and Influence. Frontiers in Psychiatry, 2018; 9(451): 01-09.

24. NEVES CFS, et al. Qualidade de vida da pessoa com esclerose múltipla e dos seus cuidadores. Revista Enermagem Referência, 2017; 4(12): 85-96.

25. PITTOCK SJ, LUCCHINETTI CF. Neuromyelitis optica and the evolving spectrum of autoimmune aquaporin-4 channelopathies: a decade later. Ann NY Acad Sci., 2016; 1366 (1): 20-39.

26. ROCHE. Mabthera®: rituximabe. Produtos Roche Químicos e Farmacêuticos S.A., 2018; 3:1-19.

27. SARVA H, et al. Diversity of Neuromyelitis Optica: Inner City Hospital Experience and Review of Literature. Journal of Neurology \& Neurophysiology, 2014; 5(2): 01-06.

28. SIMÕES M, et al. Uso de rituximabe no tratamento de linfoma linfoplasmocítico durante terapia de substituição renal contínua. Revista Brasileira de Terapia Intensiva, 2019; 31(2): 258-261.

29. TAMBUNAN L, et al. Neuromyelitis Optica Spectrum Disorder: A Case Report of Effective Combination Immunosuppressant, Corticosteroids, and Therapeutic Plasma Exchange. Open Access Macedonian Journal of Medical Sciences, 2019; 7(20): 3433-3436.

30. TRADTRANTIP L, et al. Emerging therapeutic targets for neuromyelitis optica spectrum disorder. Expert Opin Ther Targets, 2020; 2: 01-11.

31. TREBST C, et al. Update on the diagnosis and treatment of neuromyelitis optica: Recommendations of the Neuromyelitis Optica Study Group (NEMOS), 2014; 261(1): 01-16.

32. VALENTINO $\mathrm{P}$, et al. Aquaporin-4 antibody titration in NMO patients treated with rituximab. Neurology Neuroimmunology Neuroinflammation, 2016; 4(2): 01-11.

33. WOO S, et al. A Case Report of Treatment of a Patient with Neuromyelitis Optica and Suffering from Vision Disorder and Quadriplegia with Korean Traditional Medicine. The Journal Of Internal Korean Medicine, 2017; 38(5): 658-667.

34. ZAREI S, et al. Comprehensive review of neuromyelitis optica and clinical characteristics of neuromyelitis optica patients in Puerto Rico. Surgical Neurology International, 2018; 9: 242.

35. ZHANG $Y$, et al. Sexual dysfunction in patients with neuromyelitis optica spectrum disorder. Journal of Neuroimmunology, 2020; 338: 01-06. 